\title{
Interactive comment on "Pyrocumulonimbus
}

\section{Events over British Columbia in 2017: The}

\section{Long-term Transport and Radiative Impacts of}

Smoke Aerosols in the Stratosphere" by

Sampa Das et al.

Anonymous Referee \#1

Received and published: 19 February 2021

see PDF

Please also note the supplement to this comment:

https://acp.copernicus.org/preprints/acp-2020-1240/acp-2020-1240-RC1-

supplement.pdf 